Revue des patrimoines

\title{
Des vitraux par milliers... Bilan d'un inventaire : le recensement des vitraux anciens de la France
}

Françoise Gatouillat, Michel Hérold et Véronique David

\section{(2) OpenEdition}

Journals

Édition électronique

URL : http://journals.openedition.org/insitu/8884

DOI : $10.4000 /$ insitu.8884

ISSN : 1630-7305

Éditeur

Ministère de la culture

Référence électronique

Françoise Gatouillat, Michel Hérold et Véronique David, « Des vitraux par milliers... Bilan d'un inventaire : le recensement des vitraux anciens de la France », In Situ [En ligne], 6 | 2005, mis en ligne le 15 mai 2012, consulté le 01 mai 2019. URL : http://journals.openedition.org/insitu/8884 ; DOI :

10.4000/insitu.8884

Ce document a été généré automatiquement le 1 mai 2019.

\section{(i)

In Situ Revues des patrimoines est mis à disposition selon les termes de la licence Creative Commons Attribution - Pas d'Utilisation Commerciale - Pas de Modification 4.0 International. 


\title{
Des vitraux par milliers... Bilan d'un inventaire : le recensement des vitraux anciens de la France
}

\author{
Françoise Gatouillat, Michel Hérold et Véronique David
}

Qui connaissait, avant leur publication dans le Recensement des vitraux anciens de la France, le Calvaire du XVe siècle de Saint-Chef dans l'Isère, l'élégante verrière allégorique du XVIe siècle de La Saulsotte dans l'Aube ou les panneaux de la Sainte-Chapelle de Paris égarés au musée de Chalon-sur-Saône ? ${ }^{1}$ La peinture sur verre est certes en soi un objet d'étude, déjà constitué comme tel au temps des précurseurs, Félibien et Le Vieil, mais il aura fallu patienter pour en cerner l'ampleur et l'exceptionnelle fécondité : en l'absence d'un repérage systématique, comment montrer que les "villes saintes du vitrail», Chartres, Bourges, Troyes, Rouen et quelques autres ne détiennent pas de monopole? Comment alimenter de façon satisfaisante la recherche historique sur ce domaine si vaste, et comment assurer la sauvegarde de ce patrimoine particulièrement fragile ? La nécessité d'engager le Recensement des vitraux s'est imposée d'elle-même à un moment particulier de l'histoire de l'art. L'éditorial du dixième numéro de la Revue de l'Art en rappelle les principales étapes ${ }^{2}$ : au début du XXe siècle, «l'histoire du vitrail en France était entre les mains de deux peintres verriers Louis Ottin et Olivier Merson, d'un architecte, Lucien Magne, et de l'admirable historien de l'iconographie religieuse, Emile Mâle, qui ne fut pas spécialiste en la matière ${ }^{3}$. Il en résultait une sorte de doctrine unitaire, qui avait le défaut d'isoler le vitrail des autres arts et de lui prêter un développement presque autonome, ou bien de le considérer comme une technique artistique d'appoint, soumise à l'architecture, ou n'ayant pour intérêt essentiel que le déploiement des thèmes d'iconographie sacrée ». 
Figure 1

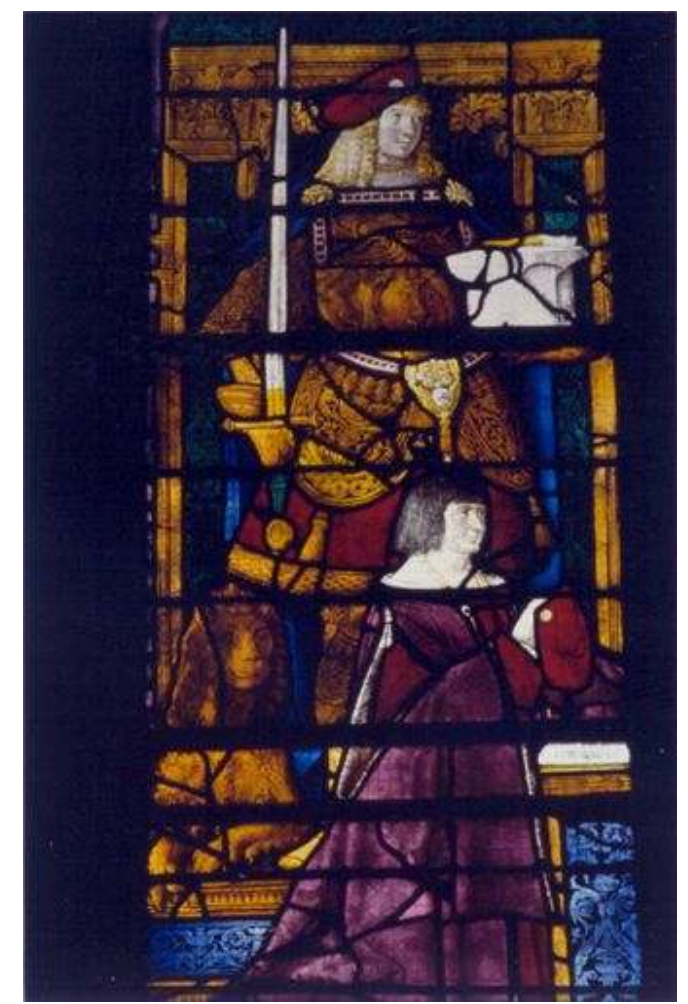

Conches-en-Ouche (Eure), église Sainte-Foy, bas-côté nord de la nef : verrière d'Arnoult de Nimègue, le donateur présenté par saint Adrien ; 1508-1510

Phot. M. Hérold (c) M. Hérold, 1995

2 Une telle situation encourageait le phénomène -il est vrai général- d'une compartimentation des «disciplines ». L'œuvre de Jean Lafond, jalonnée de nombreuses publications - de 1909 à 1973 -, a orienté la connaissance sur une toute autre voie, riche d'avenir. On doit à ce grand savant non seulement la « résurrection » d'artistes, Arnoult de Nimègue (fig. $n^{\circ} 1$ ), Romain Buron ou Jean Chastellain, mais surtout les bases des méthodes de la recherche moderne.

La campagne de dépose générale des vitraux à la veille du second conflit mondial créa des circonstances «favorables $»^{4}$ particulières, dont les spécialistes surent profiter pour donner à la recherche une impulsion décisive. Tirant les leçons de la guerre de 1914-1918, le service des Monuments historiques fit déposer préventivement en 1939 la majeure partie des vitraux classés, de même que d'autres pays européens, en particulier l'Allemagne. La surface des verrières mises à l'abri en France est estimée à plus de 50000 $\mathrm{m}^{2}$, évaluation sans doute inférieure à la réalité. Les grandes cathédrales, au même titre que des centaines d'édifices moins considérables, furent ainsi provisoirement dépouillées de leur décor vitré. C'est alors que des équipes de restaurateurs et d'historiens de l'art purent observer de façon rapprochée et donc idéale des milliers de panneaux. La constitution d'une documentation photographique cohérente et durable, faite de clichés au dixième pris systématiquement en atelier avant et après restauration afin d'établir des photomontages, fut alors développée et s'est poursuivie jusqu'aux années 1970. Sur ces nouvelles bases, la connaissance du patrimoine verrier français trouvait les moyens d'un véritable essor. Ces circonstances permirent de révéler le vitrail à l'ensemble de la 
communauté scientifique : «Il y avait là tout un chapitre de la peinture médiévale qui demandait à être intégré plus étroitement à l'histoire de l'art $»^{5}$. Avant leur repose, les œuvres les plus significatives du XIIe au XVIe siècle furent également présentées au grand public, à Rotterdam en 1952 et à Paris en 1953, où l'exposition Vitraux de France connut un vif succès ${ }^{6}$. Sous des auspices si favorables parurent des ouvrages essentiels, comme Le vitrail français, publié en 1958, travail collectif qui réunit les meilleurs spécialistes du moment ${ }^{7}$. Le phénomène n'est pas spécifique à la France, puisque l'Allemagne vit paraître en 1951 les Meisterwerke der Glasmalerei d'Hans Wentzel, et l'Italie les Vetrate italiane de Giuseppe Marchini, en 1955.

Figure 2

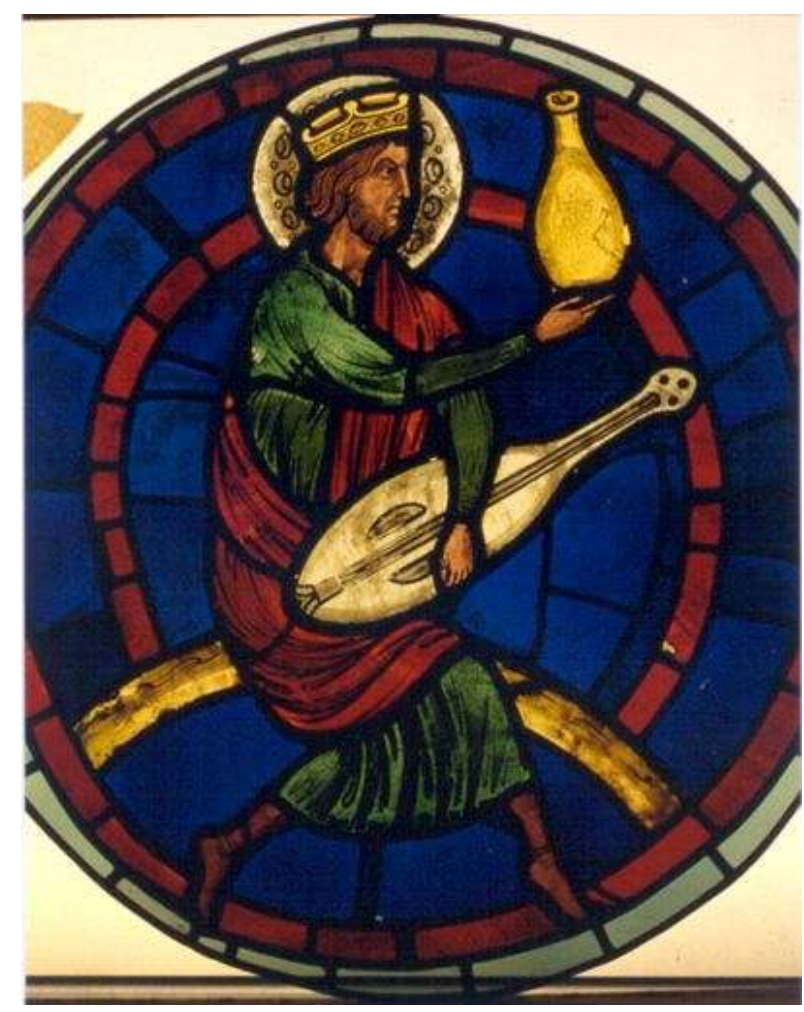

Laon (Aisne), cathédrale Notre-Dame, rose est : un vieillard de l'Apocalypse ; vers 1210

Phot. M. Hérold (c) M. Hérold

Diverses problématiques se dessinèrent alors : les liens étroits entre l'architecture et ses clôtures translucides, colorées et figurées, s'imposaient comme une évidence majeure, les historiens de l'art, notamment André Chastel, rangeant le vitrail parmi les plus belles expressions de la peinture monumentale des pays septentrionaux. Avec les travaux de Louis Grodecki, l'effort porta sur l'étude des styles, des «mains ", et donc des ateliers, ce qui n'avait intéressé ni le chanoine Delaporte dans sa grande monographie de la cathédrale de Chartres, ni Emile Mâle, qui avouait «ne pas voir en quoi les vitraux de Bourges, ceux de Laon, ou les plus anciens vitraux de Sens, de Tours et du Mans diffèrent de ceux de Chartres " (fig. $\mathbf{n}^{\circ} \mathbf{2}^{8}$. Il apparut aux auteurs du Vitrail français que leur domaine, jusque-là considéré comme un art mineur, devait être étudié non plus globalement mais en détail, répondant d'ailleurs en cela à l'évolution générale des sciences humaines. La règle devait désormais être l'approche directe, l'étude s'appuyant 
sur une critique d'authenticité exigeante, sur une «archéologie de l'œuvre » dont Jean Lafond avait été le pionnier9.

Figure 3

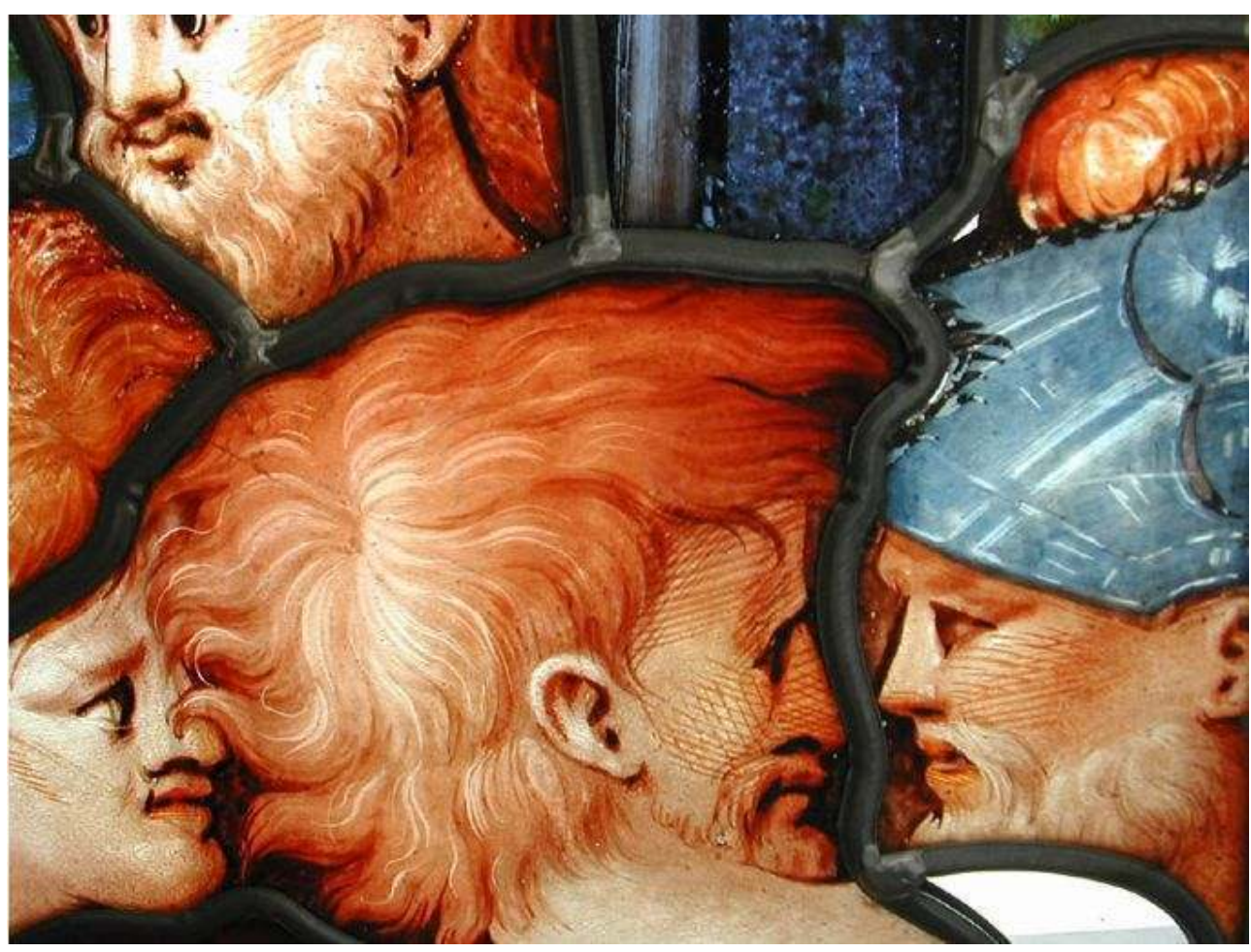

Brie-Comte-Robert (Seine-et-Marne), église Saint-Etienne, verrière de saint Jean-Baptiste, détail : grisaille rousse, verre bleu gravé ; vers 1535

Phot. G.-M. Leproux @ C G.-M. Leproux, 2003

5 De cette analyse intime devait découler un intérêt grandissant pour les questions techniques (fig. $\mathrm{n}^{\circ} 3$ ) et pour l'étude des altérations physiques ou chimiques. Une part importante de la recherche s'est centrée, dès les années 1950, sur la question des restaurations, celle des remèdes à trouver face à des phénomènes qui se sont intensifiés en raison de la pollution moderne, afin de mettre au point des prescriptions pour restaurer « autant qu'il est nécessaire et le moins possible ».

6 Un tel élan aboutit à la véritable fondation d'une discipline. Il conduisit les historiens du vitrail à réunir leurs forces pour créer une structure internationale ayant pour objectif l'étude exhaustive des vitraux anciens, le Corpus vitrearum medii aevi, dont la naissance fut formalisée en 1952 lors du congrès du Comité international d'Histoire de l'Art. Dès 1954, les première Directives furent rédigées dans le but d'assurer autant que possible l'homogénéité des publications à venir, appelées à constituer une collection. Quinze volumes étaient initialement envisagés pour l'Allemagne de l'Ouest, huit pour l'Autriche, six pour l'Espagne, etc. La France, pays le plus riche au monde en vitraux anciens, en prévoyait vingt-cinq. Mais vingt ans après la naissance du Corpus, le bilan général apparut mitigé : chaque pays avait structuré son comité selon ses moyens et adapté les normes; onze volumes étaient parus mais deux seulement en France ${ }^{10}$. A ce rythme, comment imaginer atteindre à échéance raisonnable les buts fixés? Les responsables français, conscients du défi à relever, présentèrent en 1971 au congrès de Florence le projet d'un inventaire national sommaire. 
Figure 4

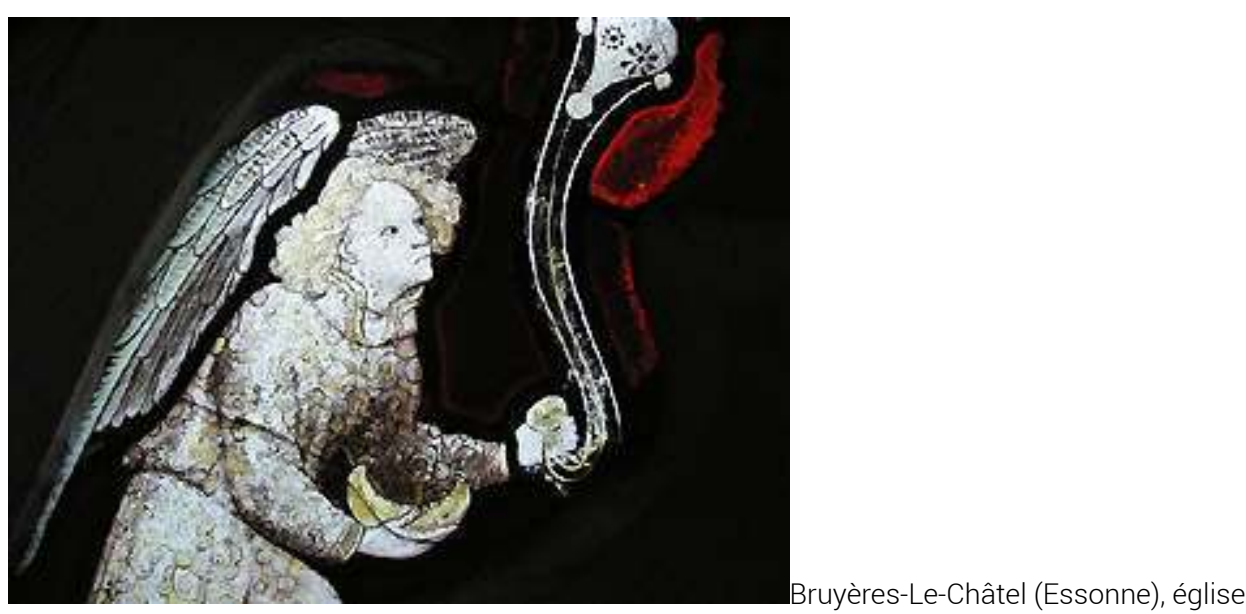

Saint-Didier, rose de la baie est de la chapelle seigneuriale : un ange thuriféraire ; vers 1420

Phot. M. Blanc-Garin (c) M. Blanc-Garin, 2004

7 La nouvelle entreprise prit le nom de Recensement des vitraux anciens de la France ${ }^{11}$, confiée à la future "Cellule vitrail» de l'Inventaire général, première équipe de recherche thématique du service et élément fort, dès sa création en 1986, du Laboratoire de recherche sur le patrimoine français (UMR 22, unité mixte Ministère de la Culture /CNRS) ${ }^{12}$. L'objectif visé est clair : établir région par région l'inventaire rapide mais complet des vitraux antérieurs à la Révolution, en somme forger un outil de travail qui repose à la fois sur un état de la bibliographie et sur une observation des œuvres in situ ou, quand l'occasion s'en présente, en atelier. Tout en préparant les travaux monographiques du Corpus, l'entreprise a pour double mission de conserver la mémoire d'un patrimoine fragile et de créer un instrument de recherche pour des publics que l'on imagine variés : historiens et historiens de l'art, iconographes, peintres verriers et maîtres d'ouvrage chargés des restaurations, partenaires patrimoniaux des diverses régions ou simples amateurs. Sa grande originalité est de prendre en compte systématiquement et sans aucune hiérarchisation les verrières religieuses, celles des édifices civils, des musées, et, si possible des collections privées. Les notices obtenues forment une collection documentaire exceptionnelle, dévoilant peu à peu une immense terra incognita (fig. $\left.n^{\circ} 4\right)$. 
Figure 5

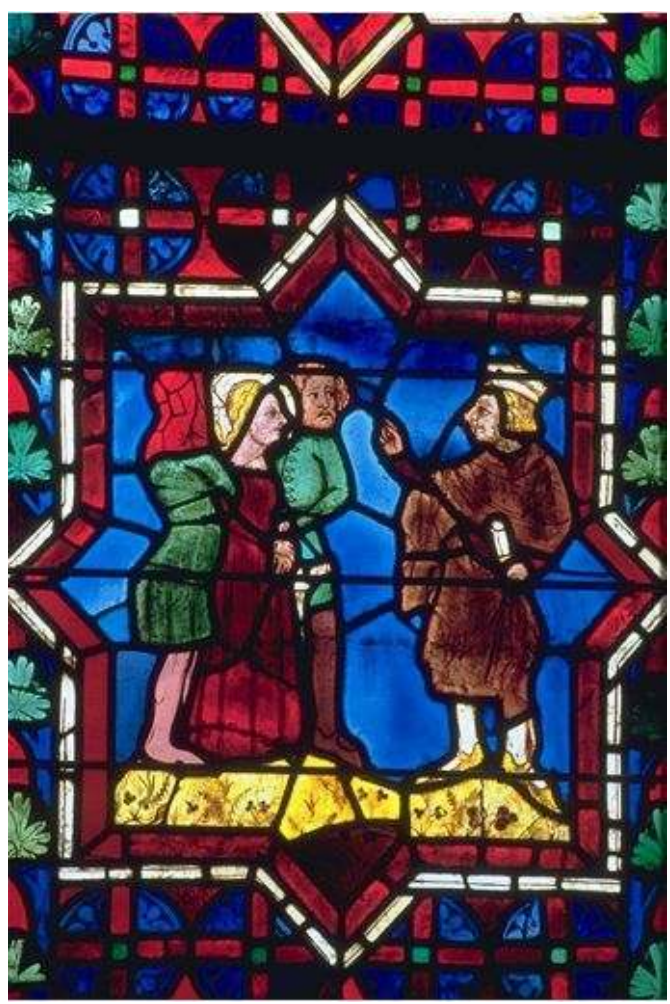

Dol-de-Bretagne (IIle-et-Vilaine), cathédrale Saint-Samson, maîtresse-vitre (1290-1300) : I'arrestation de sainte Marguerite, scène refaite à la fin du XIV siècle

Phot. Inv. B. Bègne (c) Inventaire général, ADAGP, 1999

Pour des raisons matérielles et techniques évidentes, les vitraux semblent souvent inaccessibles à l'œil et demandent un effort pour être compris. Le travail de recensement doit contribuer à surmonter ces barrières en forgeant un outil apte à guider le regard. La démarche est identique à l'analyse stratigraphique en archéologie et commence par quelques questions simples : la verrière est-elle demeurée à son emplacement primitif ? Si c'est bien le cas, sa conception concorde-t-elle avec celle de son cadre architectural, qui peut lui-même fournir des repères intéressants et vice versa? Est-elle homogène ou composite? L'agencement des panneaux, celui des scènes, semble-t-il logique, ou la composition originale a-t-elle été altérée lors d'un remontage? Une date portée a-t-elle été correctement lue par ceux qui l'ont relevée précédemment? Se rapporte-t-elle à l'œuvre elle-même ou seulement à l'une de ses parties constituantes, ou encore à l'une de ses restaurations? Les bénéfices de cette approche circonspecte peuvent facilement être mis en évidence. Parmi bien d'autres mises au point, on mentionnera la date de 1517 de la grande verrière de l'église d'Ergué-Gabéric (Finistère), habituellement lue 1571 et ainsi portée sur l'arrêté de classement, ou l'attribution d'un grand remaniement de la maîtresse-vitre du XIIIe siècle de la cathédrale de Dol à un praticien des années 1380-1400 (fig. $\mathbf{n}^{\circ}$ 5). Les pièges sont souvent bien plus redoutables. Le vitrail étant une sorte de puzzle adaptable et mobile, les résultats des «arrangements" réalisés en diverses circonstances apparaissent quelquefois particulièrement déroutants. En Bretagne encore, où l'opération de recensement s'achève aujourd'hui, les vitraux de Saint-Armel de Ploërmel offrent une disposition qui n'est pas sans surprendre. Posés depuis 1480 jusqu'au milieu du XVIe siècle, ils subirent des restaurations considérables dès les années 
1570 et surtout autour de 1600 . Cette stratification, déjà complexe mais somme toute " classique ", fut totalement bouleversée à la suite des bombardements de 1944. Le parti de restauration choisi après-guerre fut de regrouper les éléments sauvés du désastre et de les compléter sur le mode contemporain. Au terme des travaux effectués entre 1955 et 1973 par l'atelier Jacques Bony, plusieurs des fenêtres du bas-côté nord rassemblent des vestiges de leur décor primitif et ceux de verrières supprimées: dans la nef, une Pentecôte s'emboîte ainsi dans une Dormition de la Vierge, sans rapport avec la figure du donateur en place, mêlant des parties de différentes époques du XVIe siècle avec d'autres du XVIIe et du XIXe siècle, le tout complété par les inventions du peintre verrier du XXe siècle. Seules les descriptions et photographies antérieures à la guerre confrontées pas à pas aux vitraux en place aujourd'hui peuvent permettre de les déchiffrer.

Figure 6

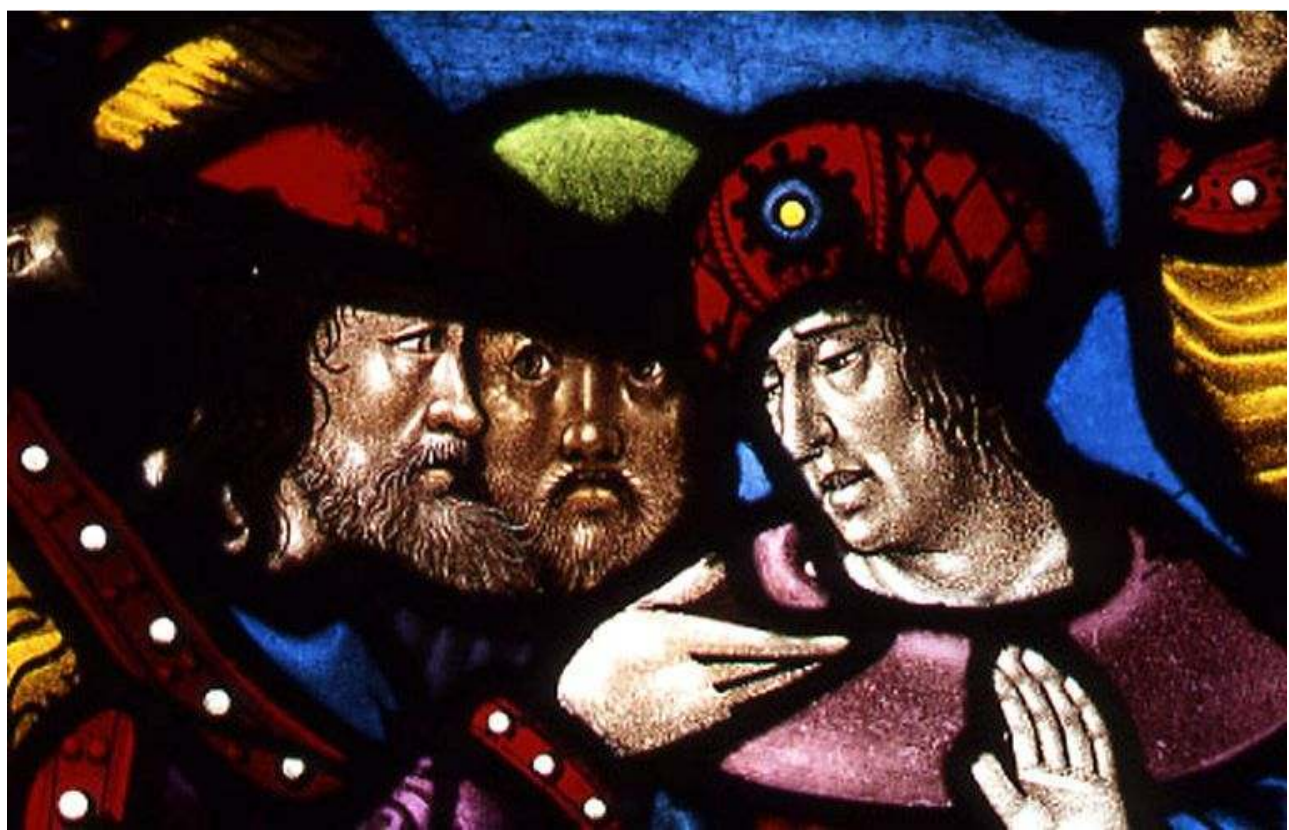

Troyes (Aube), église Sainte-Madeleine, vie de saint Marie-Madeleine, détail ; 1506 Phot. Inv. J. Philippot ( Inventaire général, ADAGP, 2005

La documentation réunie à l'occasion du Recensement sera vraisemblablement amenée à jouer le même rôle. Dans l'immédiat et pour le futur, elle livre une masse d'informations immense sur l'étendue et le contenu du corpus verrier de la France. Les dossiers établis sont consultables dans les Services régionaux d'Inventaire et, pour la partie informatisée, dans la base Palissy du Ministère de la Culture ${ }^{13}$. C'est là un outil de repérage essentiel, associé aux remarquables fonds de clichés établis par les photographes du service. Depuis 1980 , cette couverture photographique de grande qualité se veut exhaustive. De très nombreuses œuvres jusqu'alors absentes des fonds publics, la totalité des verrières de chacune des régions étudiées, sont désormais signalées et leurs images sont disponibles. On peut voir dans ce résultat une sorte d'acte de (re)naissance d'un grand nombre de vitraux auparavant ignorés car inaccessibles (fig. $\mathbf{n}^{\circ} \mathbf{6}$ ). 


\section{Des chiffres et des lieux}

Louis Grodecki avait conçu les travaux du Recensement comme un moyen d'évaluer l'ampleur du patrimoine verrier. Trente ans après l'annonce de cette nécessité primordiale, il est possible d'affirmer que l'objectif est en mesure d'être atteint, voire dépassé. La collection du Recensement comptera bientôt huit volumes ${ }^{14}$; elle est attendue à l'étranger, où les spécialistes suivent la progressive « révélation » des vitraux français au rythme des parutions ${ }^{15}$, et elle y a été imitée ${ }^{16}$. L'estimation du nombre des vitraux à recenser dans les sept régions pour l'instant inexplorées, bien moins riches que celles du nord de la France, laisse espérer l'achèvement de l'entreprise avant dix ans ${ }^{17}$. Aucun autre inventaire thématique n'aura été mené en si peu de temps. Ce qui a été étudié dans la moitié septentrionale du pays et en Rhône-Alpes correspond à au moins 80 \% de la totalité des vitraux anciens, ce qui n'est pas sans appeler quelques réflexions.

Figure 7

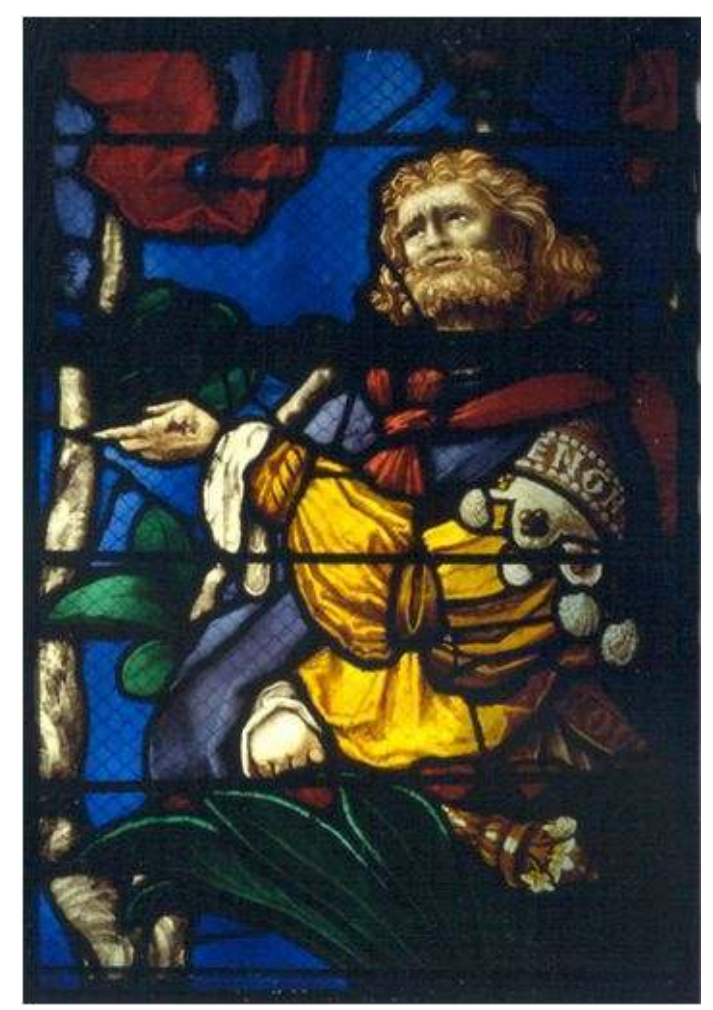

Beauvais (Oise), église Saint-Etienne : détail de l'Arbre de Jessé, par Engrand Le Prince ; 1522-1524 Phot. M. Hérold @ M. Hérold

11 Si l'incomparable richesse nationale se trouve confirmée, ces résultats montrent sa répartition très inégale dans le temps comme dans l'espace. La Champagne et la HauteNormandie sont assurément les régions les mieux dotées. Tout aléatoire que puisse être un dénombrement mettant sur un pied d'égalité une petite verrière rurale et le célèbre Arbre de Jessé d'Engrand Le Prince à Beauvais (fig. $\mathrm{n}^{\circ} 7$ ), les résultats de ce travail sont susceptibles de se traduire en chiffres permettant de répondre à quelques questions. Quelle place tiennent, par exemple, les vitraux normands dans le corpus verrier français ? La Haute-Normandie possède environ 1400 verrières antérieures à la Révolution, 
contenues dans 230 édifices, soit à peine moins que la Champagne qui en possède environ 1600. 860 de ces verrières sont du XVIe siècle, dont 750 antérieures à 1550. Le groupe normand est toutefois plus largement échelonné dans le temps que celui de la Champagne, où sur les 1160 verrières de l'Aube, département le mieux pourvu, 1042 datent du XVIe siècle ${ }^{18}$. A des degrés divers, l'écrasante majorité des verrières de la fin du Moyen Age et de la Renaissance, pressentie par Louis Grodecki, se trouve désormais pleinement confirmée et surtout précisée avec toutes ses nuances. En Bretagne, les 486 sites repérés ne contiennent que 550 œuvres, presque toutes du XVe et du XVIe siècle. On constate ici, à quelques exceptions près, l'existence de petits ensembles, qui se déclinent souvent en une seule verrière, en général la maîtresse-vitre, consacrée à l'« affichage " d'une hiérarchie nobiliaire ici exceptionnellement complexe. Dans d'autres régions encore à étudier, comme en Poitou-Charentes, certains départements sont presque entièrement dépourvus de vitraux anciens : pourquoi de tels vides?

\section{Du bénéfice de tout voir}

12 «Tout voir» en peu de temps dans un espace donné est un privilège qui procure de nombreux avantages. C'est là une arme décisive pour retrouver les liens perdus entre les œuvres démembrées, pour vérifier des attributions ou en proposer de nouvelles, pour construire des chronologies relatives. A l'exploration systématique d'un patrimoine appartient aussi le plaisir de la découverte, car les œuvres inédites ne manquent pas.

Figure 8

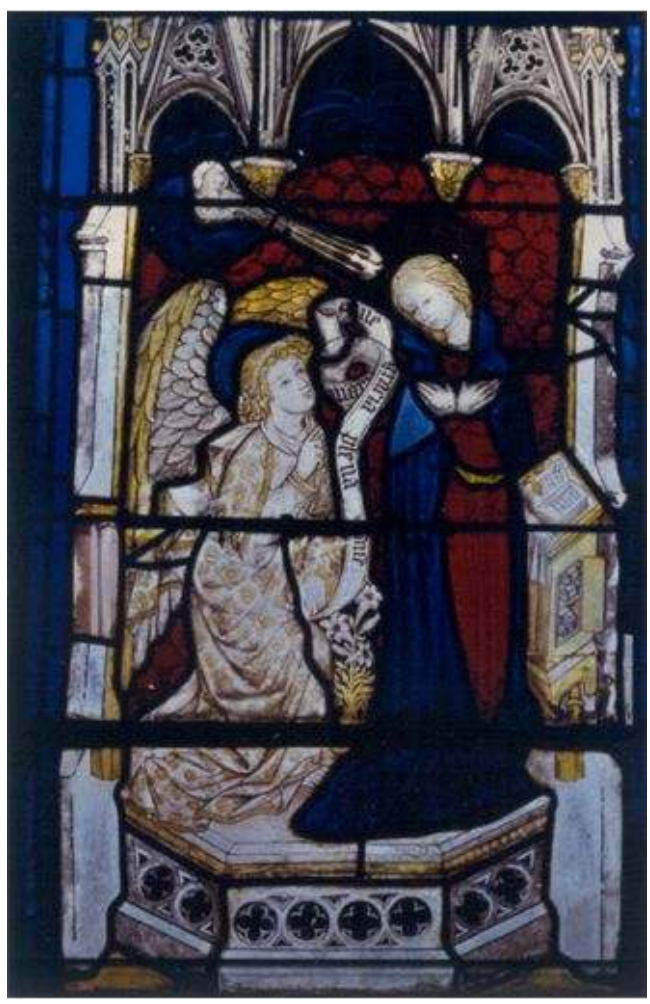

Chastellux-sur-Cure (Yonne), oratoire du château de Chastellux : I'Annonciation ; vers 1415

Phot. Inv. P. Garrigue @ Inventaire général, ADAGP, 1982 
Dans l'Yonne, par exemple, les panneaux ornementaux de la fin du XIIe siècle de la nef de l'église de Cudot, proches des vitreries en usage dans les édifices cisterciens, n'avaient jamais été remarqués ${ }^{19}$; comme eux, la verrière de l'oratoire du château médiéval de Chastellux-sur-Cure, aménagé en 1415 pour le maréchal de France Claude de Beauvoir a pu être étudiée et photographiée pour la première fois à la faveur du Recensement ${ }^{20}$ (fig. $\mathbf{n}^{\circ}$ 8). La mémoire accumulée au fil de l'avancement des travaux est évidemment favorable au jeu de l'identification d'œuvres conservées hors contexte.

Figure 9

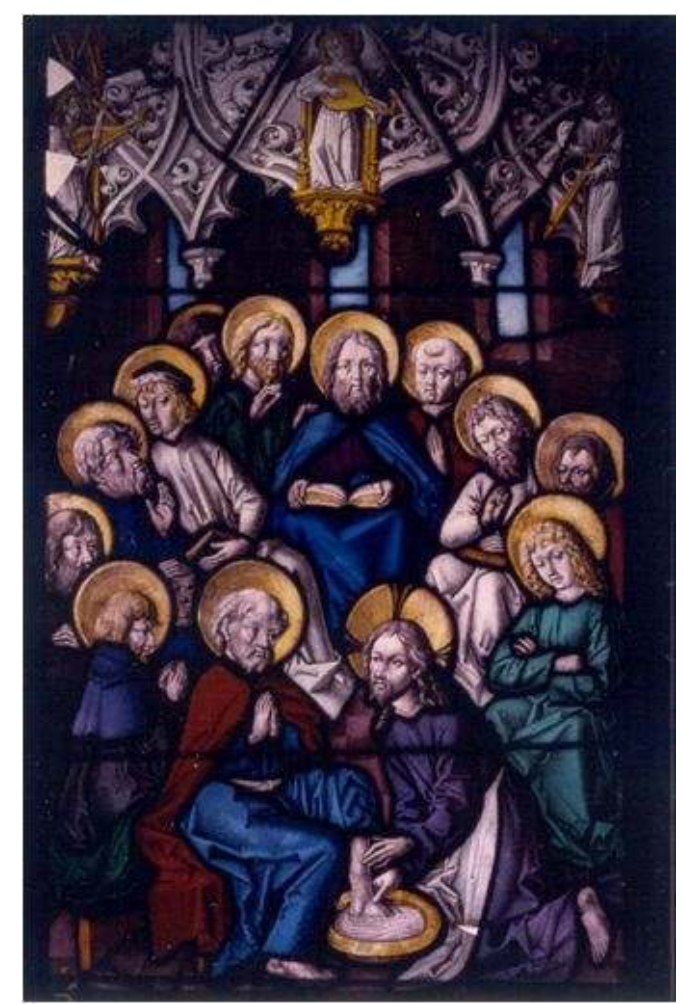

Strasbourg (Bas-Rhin), Musée de l'Oeuvre Notre-Dame : le Lavement des pieds, provenant de l'église Sainte-Madeleine ; vers 1475-1480

PHOT. M. HÉROLD ㄷ M. HÉROLD, 1994

Des panneaux alsaciens du XVe siècle ont ainsi pu être retrouvés hors région, l'un provenant de l'église Sainte-Madeleine de Strasbourg (fig. $\mathbf{n}^{\circ}$ 9), un autre de SaintGeorges de Sélestat ${ }^{21}$. Ceux du début du XVIe siècle examinés dans la chapelle du château de Lux (Côte-d'Or) paraissaient bien avoir une forte « saveur parisienne "; ils ont en effet été soustraits aux fenêtres de la nef de l'église Saint-Merry au milieu du XVIIIe siècle, ce qu'il a été possible de prouver ensuite ${ }^{22}$. L'une des dernières découvertes permises par le Recensement est bretonne. La collection réunie au château de Rochefort-en-Terre (Morbihan) par le peintre américain Alfred Klots contient un ange musicien provenant de l'une des bordures des verrières réalisées pour la chapelle d'axe de la cathédrale de Rouen vers 1310. Malgré la distance qui le sépare de son lieu d'origine, la proposition avancée repose sur la connaissance des compositions équivalentes restées en place, tirées de la même table de verrier ou du même patron. Les collections privées comme les musées et, parfois, les dépôts des ateliers de restauration constituent le terrain privilégié de telles découvertes. 
Figure 10

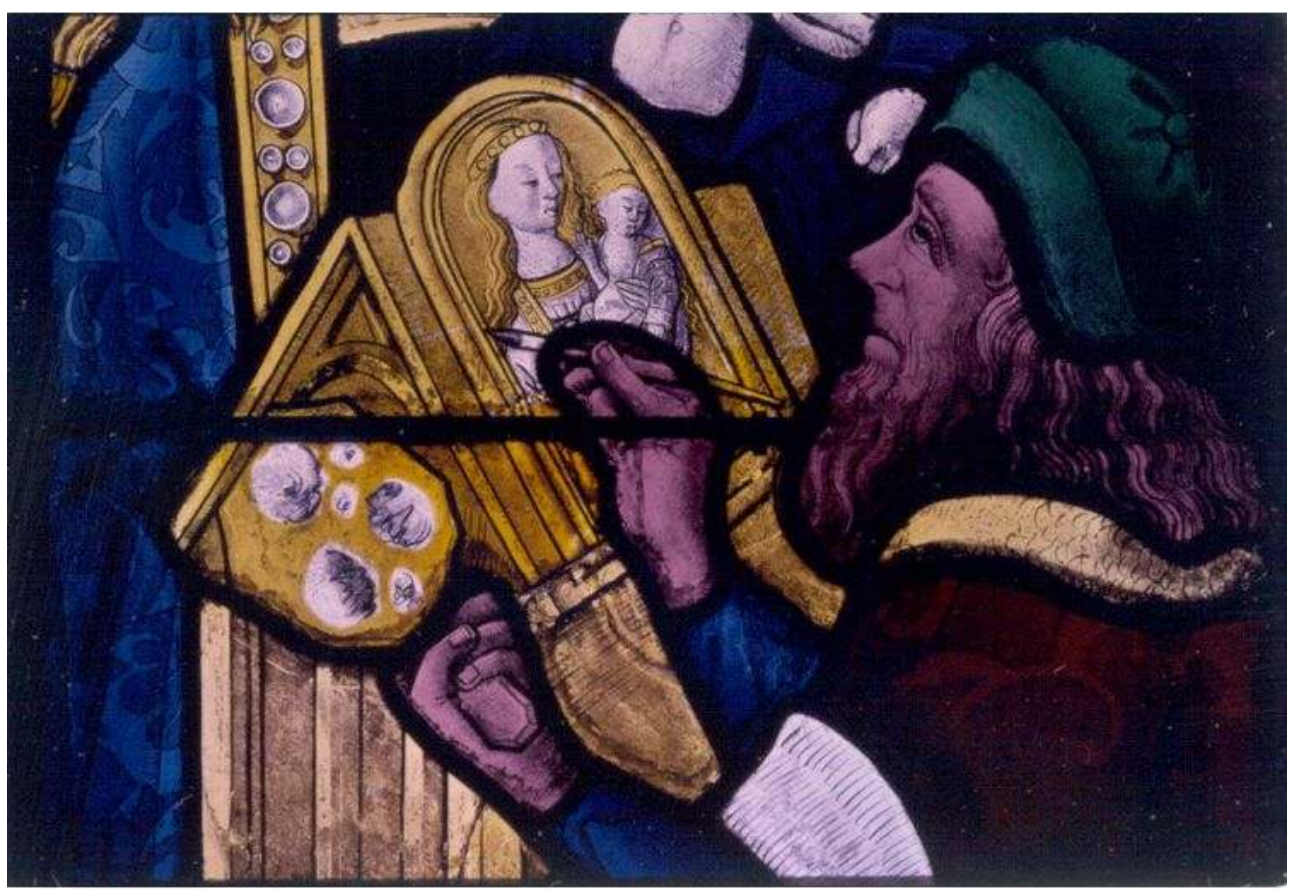

Evreux (Eure), cathédrale Notre-Dame, chapelle d'axe : saint Luc peintre de la Vierge à l'Enfant ; 1467-1469

PHOT. M. HÉROLD @ M. HÉROLD, 2001

Le privilège de « tout voir » conduit naturellement à réviser de nombreuses datations. Les œuvres ayant retrouvé leur place dans un contexte plus général bénéficient d'une chronologie relative affinée. Ainsi le décor vitré de la chapelle d'axe et du bras sud du transept de la cathédrale d'Evreux, réalisé sur ordre du roi Louis XI avant 1470, peut désormais être attribué à deux ateliers locaux intervenant en collaboration avec un troisième venu de Rouen (fig. $\mathbf{n}^{\circ} \mathbf{1 0}$ ). Cette référence acquise, des verrières de SeineMaritime apparentées, jusqu'ici considérées sommairement comme de la fin du XVe voire du XVIe siècle, à Saint-Jean d'Elbeuf et à Vatteville-la-Rue, ont pu être datées de la même décennie.

16 A l'échelle d'une ville d'une particulière importance, comme Rouen à la fin du Moyen Age et au temps de la Renaissance, étudier la totalité des vitraux conservés permet de réviser sur le mode d'une plus grande complexité ce qui pouvait être considéré comme acquis. Même sans avoir exploité de façon approfondie les immenses ressources offertes par les archives, la notion traditionnelle d'école régionale résiste peu à l'observation des œuvres, tout comme la place attribuée aux " grands artistes ». Il en est ainsi du peintre et peintre verrier d'origine néerlandaise Arnoult de Nimègue, actif dans la ville entre 1503 et 1513. En « ressuscitant » cette personnalité, Jean Lafond affirme qu'elle a orienté « (...) toute la production rouennaise pendant un quart de siècle ${ }^{23}$. Arnoult est assurément l'auteur de très grands chefs-d'œuvre, l'Arbre de Jessé de l'église Saint-Godard à Rouen ou le vitrail de Guillaume Toustain de Frontebosc à Conches-en-Ouche (Eure) (voir fig. $\mathbf{n}^{\circ} \mathbf{1}$ ). Par la qualité exceptionnelle de ses œuvres, saisissante au regard de toute la production contemporaine, la valeur hors pair de cet artiste flamand peut être reconnue aisément. Cependant, le fait même d'isoler une individualité dont les œuvres sont signées ou bien documentées ne suffit pas à caractériser un centre artistique aussi fécond. A Rouen, dans 
les premières années du XVIe siècle, Arnoult domine-t-il réellement la production locale en remportant les marchés lancés par les donateurs les plus considérables ou influents, dans les édifices majeurs de la ville ou de la région? Ce n'est pas le cas. Il a travaillé à Saint-Ouen de Rouen et pour l'abbatiale de Fécamp au service d'Antoine Bohier, mais est absent des plus grands chantiers du moment, en particulier de ceux du cardinal d'Amboise. Au palais archiépiscopal et à la cathédrale de Rouen règne Jean Barbe, représentant d'une importante dynastie de peintres verriers locaux; celui-ci travaille aussi pour Gaillon, où les commandes les plus notables reviennent à Antoine Chennesson d'Orléans, dont le choix relève vraisemblablement du réseau des artistes protégés par la famille d'Amboise. Ajuste titre reconnu comme l'un des plus grands maîtres de la première Renaissance, Arnoult de Nimègue ne semble pas avoir bénéficié d'une situation privilégiée dans le milieu rouennais au début du XVIe siècle. En l'absence de réglementation corporative, aucun obstacle juridique ne s'oppose à l'installation dans la ville d'artistes étrangers et à l'importation d'œuvres. Arnoult peut être considéré sans trop de risque d'erreur comme le plus parfait représentant du courant nordique dans la capitale de la Normandie, mais n'est assurément pas le seul venu des anciens Pays-Bas.

Figure 11

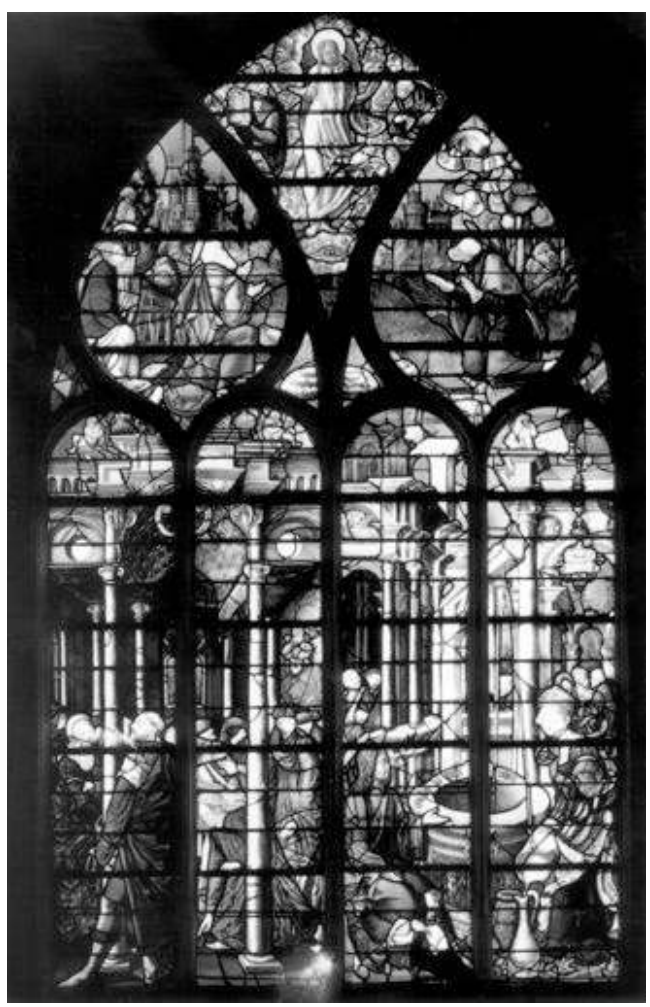

Caudebec-en-Caux (Seine-Maritime), église Notre-Dame, bas-côté sud de la nef : verrière de la Femme adultère, de la Samaritaine au puits et de la Transfiguration ; 1532

Phot. Inv. T. Leroy @ Inventaire général, ADAGP, 1993

17 L'auteur du curieux vitrail de Job de l'église Saint-Patrice s'affiche comme bruxellois ${ }^{24}$; le peintre verrier -rouennais ?- du remarquable Jugement dernier de l'église Saint-Vincent, exposé au musée des Beaux-Arts de Rouen en $1995^{25}$, pourrait avoir eu en main des dessins de Colyn de Coter, dont il se montre si proche. L'auteur du carton du célèbre vitrail de la Femme adultère et de la Samaritaine à Caudebec-en-Caux (1532) (fig. $n^{\circ} 11$ ) ne se situe-t-il pas encore dans la tradition anversoise, particulièrement en vogue entre 1520 et 
1530 ? Ces artistes nordiques, dont on ne perçoit pas encore parfaitement bien le nombre ni l'importance, sont évidemment en concurrence avec des ateliers locaux, une dizaine semble-t-il à l'époque, et bien entendu avec d'autres foyers de production. Il faut également compter avec le rayonnement de Paris, si puissant au XVIe siècle et si naturel dans la grande ville qui lui est reliée par la Seine. Le phénomène a été relevé à propos des œuvres attribuées au Maître de la Vie de saint Jean-Baptiste ${ }^{26}$, puis récemment enrichi par les études consacrées au peintre parisien Gauthier de Campes, dont plusieurs cartons ont été interprétés à Rouen pour l'église paroissiale Saint-Etienne-la-Grande-Eglise et pour Saint-Ouen de Pont-Audemer ${ }^{27}$. Le travail de "défrichage" réalisé par le Recensement permet déjà de saisir la complexité d'un univers ici particulièrement foisonnant. Si bien des œuvres peuvent être désignées comme caractéristiques de la production locale, l'ancienne notion d'« Ecole normande ", dont on voit bien les limites, est devenue totalement obsolète. L'échelle des études pertinentes est toute autre : elle se place à l'intérieur des réseaux des commanditaires, sur les chantiers, considère les échanges artistiques par la circulation des documents graphiques, des œuvres ellesmêmes et, naturellement, des hommes.

\section{Analyse des œuvres et nouveaux repères}

La confrontation quotidienne avec le terrain est l'un des points forts du Recensement. Ses bénéfices sont multiples, particulièrement évidents lorsqu'il s'agit de l'observation des techniques anciennes, qui fait partie de l'étude de chaque verrière. Elle peut devenir un excellent outil d'analyse, à partir des repères concrets que fournissent les matériaux qui constituent le vitrail, verre, plomb, peinture. Sous cet angle, il est possible de considérer le vitrail comme le plus vaste gisement d'échantillons de verres plats datés, et donc comme une source majeure pour l'histoire de l'industrie verrière. Observer la qualité des verres, leur état de conservation, leurs dimensions, noter la « palette » en usage donnent à l'historien du vitrail des repères qui coïncident souvent aux césures formelles relevées par ailleurs. Elles sont parfois flagrantes, comme à Troyes à la fin du XVe et au début du XVIe siècle. La verrière de la Passion de l'église de la Madeleine (baie 4), posée au plus tard en 1494, souligne le passage d'un vitrail clair à un vitrail exubérant de couleurs qui prévaut jusque vers 1530 . 
Figure 12

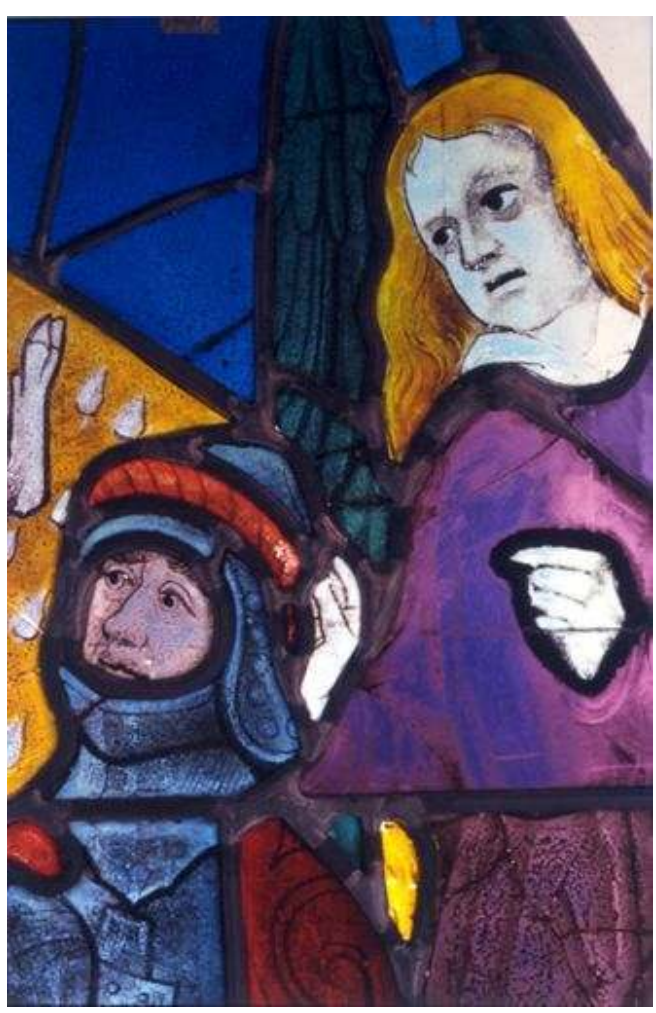

Evreux (Eure), cathédrale Notre-Dame, chapelle d'axe, Gédéon et la toison (1467) : buste de l'ange restauré vers 1530

PHOT. M. HÉROLD @ M. HÉROLD, 2000

Les verrières de Daniel et de la Passion des chapelles méridionales de l'église SaintPantaléon (baies 6 et 8) marquent en 1531 le début du succès des compositions en camaïeu, peintes sur de grandes pièces de verre blanc, transparent et fin. Les verres du XVe siècle, saturés ou blanc nacré -aujourd'hui très corrodés- se distinguent nettement de ceux du siècle suivant, réguliers, d'un bel éclat et parfaitement conservés; il a été possible de le vérifier dans la chapelle d'axe de la cathédrale d'Evreux, avec le panneau de la toison de Gédéon restauré vers 1530 (fig. $\mathbf{n}^{\circ}$ 12). La question se pose, sans réponse tranchée encore, des liens entre ces transformations esthétiques et les progrès de l'industrie verrière.

D'autres repères chronologiques qui semblaient fixés évoluent par l'observation directe des œuvres. L'apparition des émaux au XVIe siècle, longtemps interprétée comme signe de décadence par une coupable collusion avec la peinture de chevalet, était jusqu'il y a peu située au milieu du XVIe siècle, avec pour premiers exemples datés deux verrières de Montfort-l'Amaury datées de 1543 (baie 16, Enfance du Christ) et de 1544 (baie 15, Ecce homo ) comportant l'emploi d'émaux bleu et violet. Or des recherches récentes, portant en particulier sur la production germanique de la fin du Moyen Age, ont établi la présence de grisailles de couleur ou d'émaux dès les années 1435-144028. En France, la confirmation de ces usages a été constatée devant les vitraux de la Sainte-Chapelle de Chambéry (vers 1548) déposés pour restauration, et à la chapelle Saint-Pabu de Saint-Guen (Côtesd'Armor) à l'occasion du Recensement ; ce dernier ensemble, réalisé vers 1500, comporte un émail vert émeraude. Ces repères nouveaux sont évidemment destinés à évoluer au 
gré des observations futures. Quoi qu'il en soit, il apparait que l'usage de ces techniques ne transforme pas en profondeur l'art de "peindre avec la lumière ». Ces pratiques restent annexes, surtout dans le vitrail monumental. Elles n'annoncent en rien « la fin d'un art » mais illustrent les liens naturels entre arts du feu, céramique, verre creux et vitrail.

21 Avant d'être une peinture sur verre avec ses modes d'expression particuliers, le vitrail, comme tous les autres arts, prend forme par le dessin. Deux étapes graphiques déterminantes précèdent sa réalisation technique : préexiste généralement un document de référence, dessin, gravure ou autre, avant l'établissement obligé des cartons à grandeur d'exécution, d'une importance décisive pour la peinture et pour les choix de coupe. Ces documents, conservés en nombre pour les XIXe et XXe siècles, sont en revanche exceptionnels au Moyen Age et au XVIe siècle. Les rares exemplaires connus, en particulier l'extraordinaire ensemble du XVIe et du XVIIe siècle de l'église Saint-Jean de Gouda en Hollande, méritent évidemment un intérêt tout spécial mais ne peuvent être considérés comme directement transposables. Pour combler, au moins partiellement, ces lacunes dommageables, il est un moyen mis au point par la recherche moderne, qui s'appuie sur l'étude a posteriori de « séries » d'œuvres répétées, nombreuses en matière de vitrail ${ }^{29}$. Le Recensement offre naturellement le moyen le plus adapté au repérage des œuvres « apparentées » d'un corpus. L'expérience en a été faite en Champagne à la suite de Paul Biver, initiateur dès 1913 des recherches sur le "Mode d'emploi des cartons", mais aussi à Paris et en Normandie. Une fois réunie la série des œuvres comparables, plusieurs critères d'analyse leur sont appliqués, reposant sur des paramètres matériels propres au vitrail, peinture monumentale sur support translucide : la structure du réseau des plombs, le positionnement des données principales du dessin, la coloration, les options prises dans l'utilisation de la peinture. Or la correspondance ou l'absence de l'un, de plusieurs, ou de tous ces paramètres revêt une signification précise. Leur coïncidence générale désigne des œuvres tirées d'un même patron et réalisées par le même atelier; les disparités des données picturales et la non-coïncidence des réseaux des plombs désignent en revanche des copies réalisées par des interprètes différents. Cette « machine à remonter le temps » adaptée au vitrail offre des bénéfices dont l'intérêt est évident : distinguer ce qui revient au peintre cartonnier et au peintre verrier, désigner les œuvres tirées d'un même carton et les isoler de celles qui leur sont seulement apparentées permet de clarifier bien des points essentiels pour saisir l'articulation d'une production à première vue homogène. Bien des mises au point deviennent ainsi possibles, fondées sur des données matérielles indiscutables. Ainsi les œuvres normandes tirées de cartons d'un peintre parisien, le Maître d'Anne de Bretagne, ont-elle pu être distinguées de leurs équivalents parisiens et de leurs diverses imitations (fig. $\left.\mathbf{n}^{\circ}{ }^{13}\right)\left(\right.$ fig. $\left.^{\circ}{ }^{\circ} 14\right)$. 
Figure 13

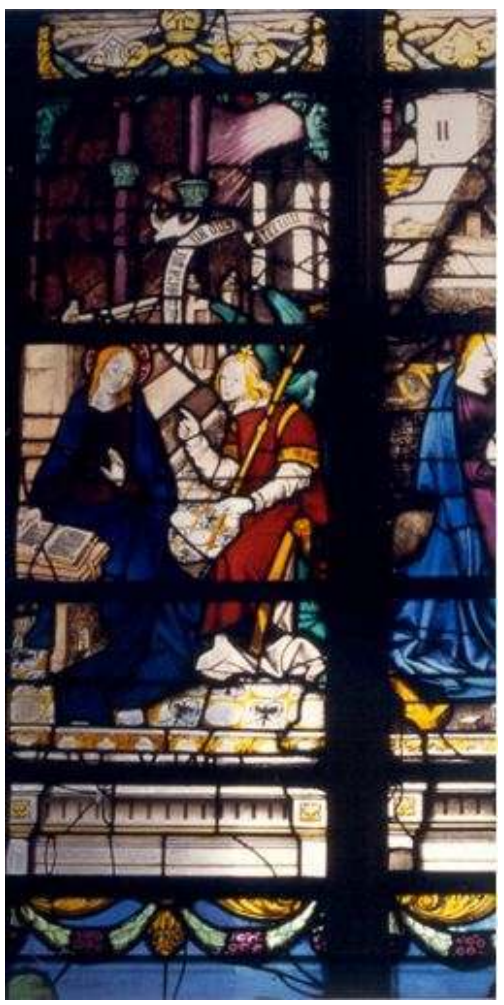

Rouen (Seine-Maritime), église Saint-Godard : I'Annonciation, début du XVIe s., scènes tirées de modèles du Maître des très petites Heures d'Anne de Bretagne

PHOT. M. HÉROLD @ M. HÉROLD 
Figure 14

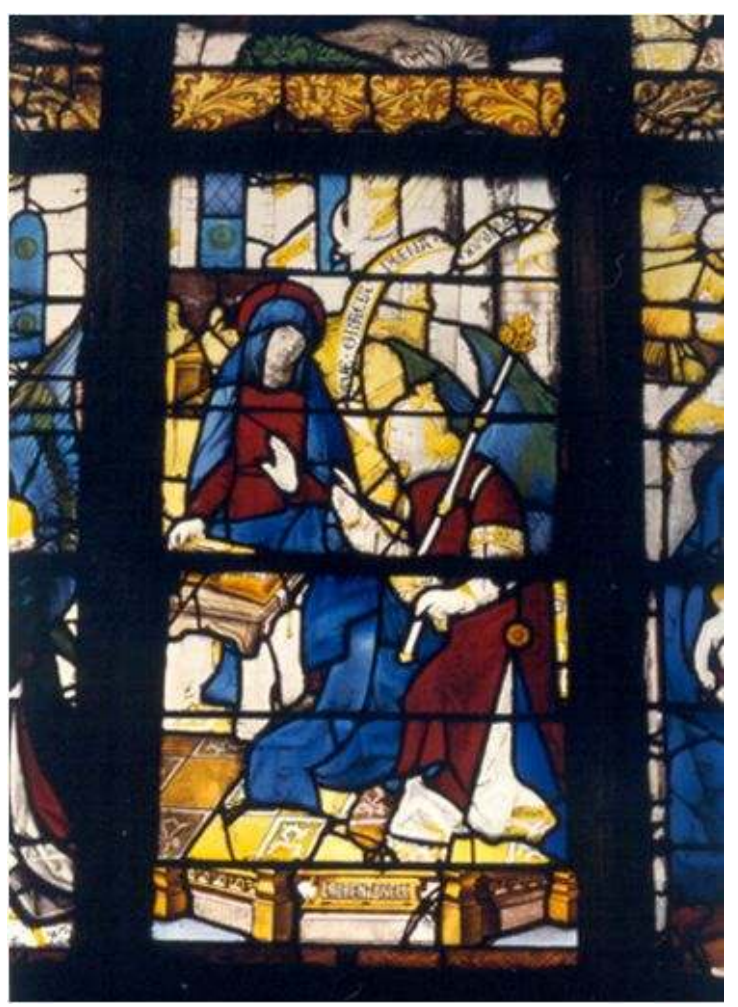

Paris, église Saint-Etienne du Mont : I'Annonciation, début du XVIe S., scènes tirées de modèles du Maître des très petites Heures d'Anne de Bretagne

Phot. M. Hérold @ M. HéroldSans ces méthodes qui s'appuient à rebours sur le contenu même des documents graphiques en usage, de telles questions ne pourraient progresser. Elles sont d'ailleurs adaptables et ont été pratiquées avec des objectifs proches dans l'étude d'autres domaines de l'activité artistique qui requièrent l'emploi de documents à grandeur d'exécution, comme la tapisserie ou la peinture murale ${ }^{30}$, pour lesquels les artistes pourvoyeurs de modèles sont d'ailleurs parfois les mêmes.

Ces exemples montrent de quelle façon se construit un échange permanent entre les données brutes livrées par le Recensement et les modes d'analyse qu'elles permettent et suscitent. Chacune des publications qui concluent les opérations régionales apporte donc une matière nouvelle et des résultats en constant progrès. Les volumes du Recensement des vitraux comprennent les notices individuelles des œuvres étudiées. Le matériau de toute autre étude se trouve ici, déjà passé au crible de la critique historique, et surtout de l'approche archéologique qui le rend praticable. La collection des notices alimente les textes introductifs, devenus plus ambitieux au fur et à mesure de l'expérience acquise. Ces introductions, qui forment dans bien des cas les toutes premières synthèses à l'échelle régionale, constituent aussi de véritables outils destinés à guider le chercheur dans l'océan des œuvres recensées. La chronologie critique proposée est sur ce point déterminante, illustrée étape par étape par les parentés techniques, iconographiques et stylistiques identifiées. Ces textes marquent une étape assurément décisive pour la recherche, à la fois bilan des connaissances réunies et passerelle vers l'avenir. 


\section{L'élargissement des champs d'action du recensement}

Dépassant le cadre chronologique qui lui était fixé, le champ d'action historique du Recensement s'est naturellement élargi aux XIXe et XXe siècles.

Figure 15

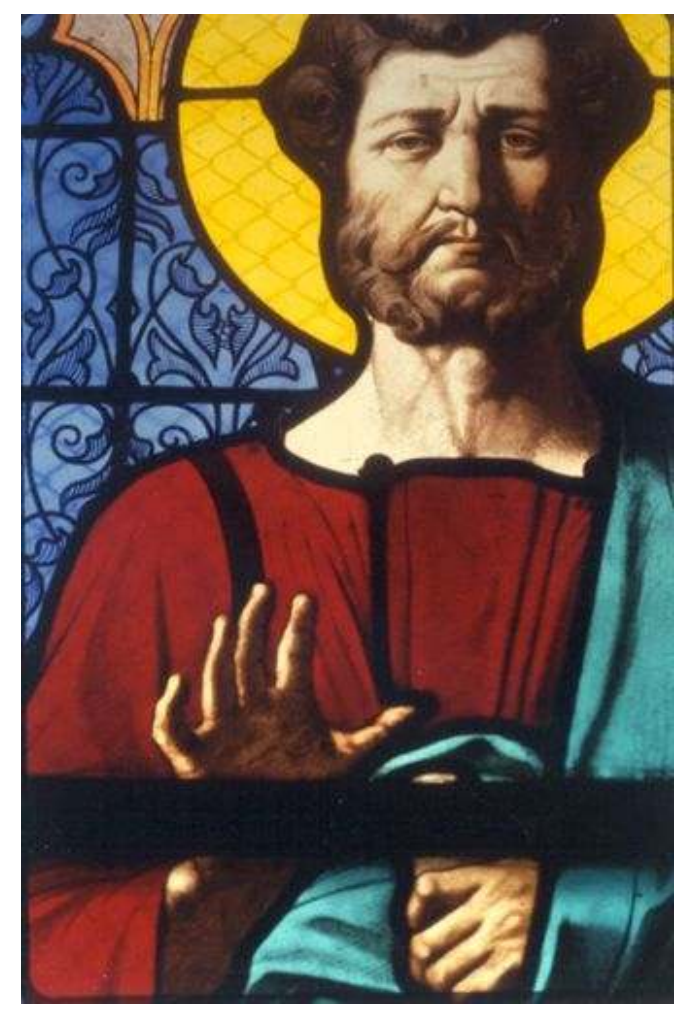

Paris, chapelle Notre-Dame de la Compassion : saint Philippe sous les traits du roi Louis-Philippe, par la Manufacture de Sèvres sur des cartons de Jean-Auguste-Dominique Ingres

Phot. F. Gatouillat (c) F. Gatouillat, 1980

L'entreprise était dès l'origine sensibilisée aux «vitraux d'exception» auxquels s'attachaient de grands noms, Ingres (fig. $n^{\circ} 15$ ), Viollet-le-Duc ou Chagall ${ }^{31}$. Mais c'est en réalité au cours de ses travaux "au jour le jour", qui requièrent l'analyse des restaurations et des compléments apportés aux vitraux anciens, que la Cellule vitrail s'est familiarisée avec la production des ateliers modernes. Celle-ci n'est d'ailleurs pas sans rapports avec celle de leurs devanciers si l'on songe aux « répliques » de verrières du XVIe siècle de Conches, Bernay et Caudebec signées des normands Duhamel-Marette et Boulanger, par exemple à Verneuil, au Vaudreuil, à Quillebeuf et à Fécamp, ou aux scènes que Gsell a empruntées à une verrière de Ferrières-en-Gâtinais pour compléter la Vie de la Vierge de Saint-Godard de Rouen. Du repérage méthodique de très nombreuses signatures est né le Répertoire des peintres verriers du XIXe siècle ${ }^{32}$, rapidement transformé en un formidable outil de travail qui établit la généalogie de chacun des ateliers jusqu'à l'époque contemporaine, dévoilant ainsi leurs multiples ramifications : qui savait Félix Gaudin le triple héritier des fonds Thibaud et Thévenot à Clermont-Ferrand et d'Oudinot à Paris? Comment dater une verrière de l'un des Champigneulle sans connaître l'histoire complexe de cette dynastie qui engendra des maisons rivales à Bar-le-Duc et à Paris? 
Cette liste est en perpétuelle évolution, au fur et à mesure des terrains parcourus par les équipes de l'Inventaire comme au fil des découvertes des " gisements » documentaires, archives d'ateliers, cartons, etc.

Figure 16

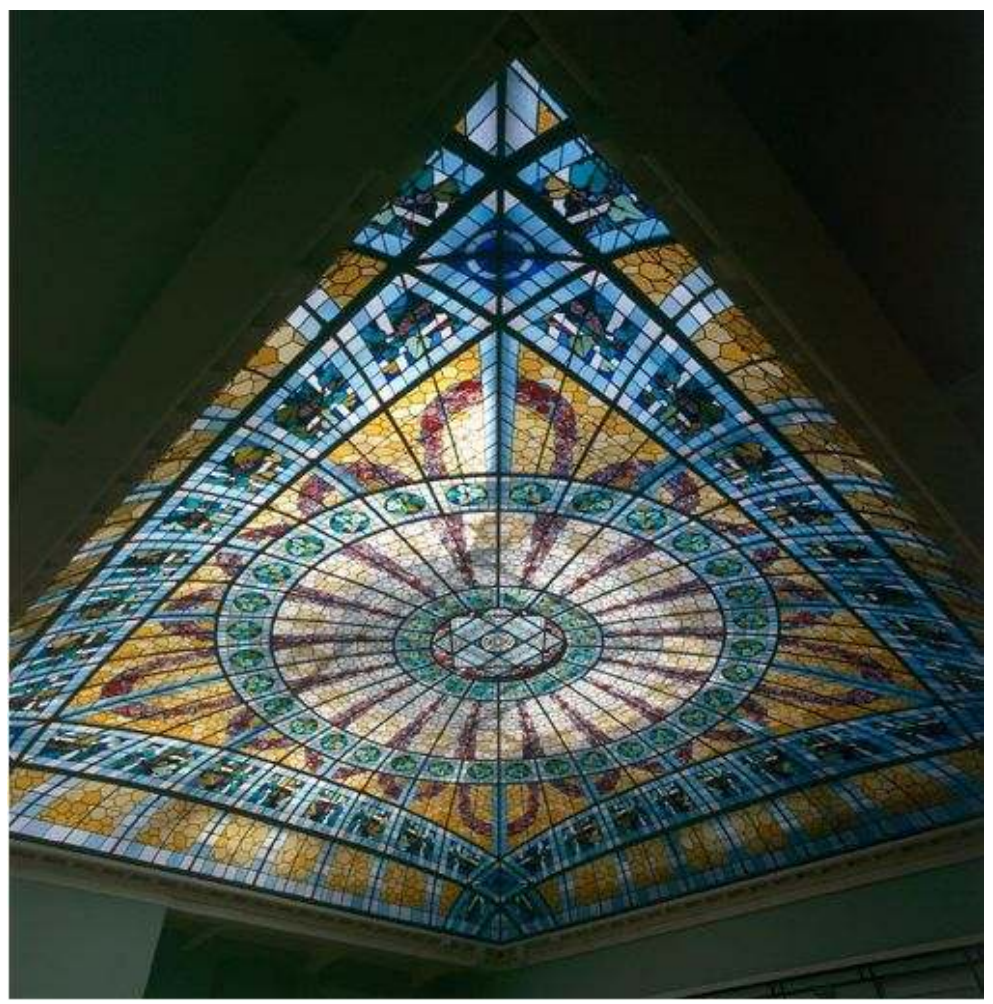

Reims (Marne), la coupole de l'ancien magasin de mode « Au petit Paris », par Jacques Simon, 1923 Phot. Inv. J. Philippot (C) Inventaire général, ADAGP, 2000

Outre le développement de ce répertoire, bientôt intégré au Thesaurus des Auteurs ${ }^{33}$ actuellement mis au point par le bureau de la méthodologie de l'Inventaire, le rôle de la Cellule vitrail dans la recherche sur ces périodes est déterminé par la nature même du patrimoine verrier. En transposant l'analyse de Claude Mignot sur l'architecture ${ }^{34}$, on peut affirmer que la production des XIXe et XXe siècles apparaît d'abord comme un phénomène quantitatif. Rares sont les communes de France qui ne possèdent pas au moins une verrière de ce temps, généralement à l'église mais parfois aussi dans des bâtiments civils, demeures particulières mais aussi magasins (fig. $\mathbf{n}^{\circ}$ 16), banques, cafés, salles de spectacle ou mairies (fig. $\mathbf{n}^{\circ} \mathbf{1 7}$ ). A cette abondante production s'attachent des critères tout autres que ceux qui ont induit l'étude du patrimoine ancien. De là un regard encore bien souvent dédaigneux, voire une absence de regard sur des œuvres trop familières, qui furent exclues de la dépose des vitraux classés à la veille de la Seconde Guerre mondiale. 


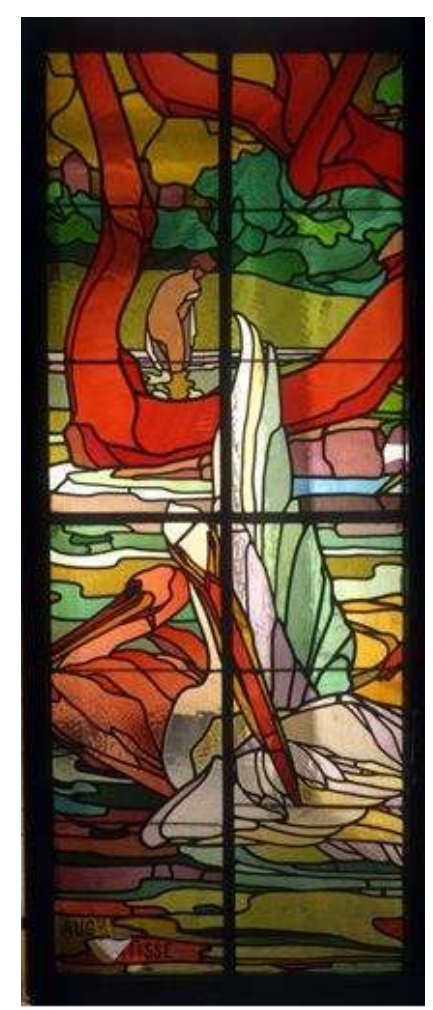

Paris, Hôtel de Ville : la verrière du Lac, par Auguste Matisse, 1924

Phot. COARC Christophe Fouin

Plus les recherches s'affinent, plus la liste des œuvres à inscrire dans le triste inventaire des disparus s'allonge: on citera, à Paris, l'ensemble vitré des murs au plafond du restaurant basque Chiquito par l'atelier Barillet en 1928, l'Assomption réalisée par Jacques Gruber à l'église Saint-Christophe de Javel en 1932, ou bien encore, à Wittenheim (HautRhin), les vitraux de l'église Sainte-Barbe de Georges Desvallières en 1930. La prise en considération de ces œuvres a certes progressé mais, aujourd'hui encore, tout danger n'est pas écarté. La création contemporaine fait encore disparaitre ou déplacer des vitraux jugés sans intérêt : les compositions de Bazaine à l'église Saint-Séverin n'ont-elles pas pris la place de celles qu'Emile Hirsch avait réalisées entre 1876 et 1899, heureusement recueillies au dépôt de Champs-sur-Marne ? Le même constat peut être fait à propos des sources en mesure d'éclairer la genèse des œuvres : des fonds d'ateliers aussi importants que ceux de Louis Barillet ou de Jacques Gruber sont aujourd'hui à peu près perdus $^{35}$. Seules de patientes recherches, essentiellement basées sur l'examen des dossiers d'œuvres, pourront servir à reconstituer l'histoire de ces structures. Par bonheur, depuis les années 1980, on assiste à un formidable développement de l'intérêt pour le XIXe siècle, bientôt imité pour le XXe siècle, de la part des communautés scientifiques ${ }^{36}$ mais aussi venant du public, qui a réellement contribué à repenser la vision de ce patrimoine ${ }^{37}$. L'attention a été attirée par les questions les plus variées, d'ordre économique et social, comme l'organisation de la production d'une entreprise (cinquante ouvriers chez Max Ingrand après la Seconde Guerre mondiale, un ou deux chez Marguerite Huré), ou, parmi bien d'autres exemples, à propos de l'histoire des mentalités religieuses, comme à la cathédrale de Nevers, où l'Eglise justifia le refus du carton de Markus Lüpertz (1990) par sa vision trop désespérée de l'homme. De tous côtés, les recherches ont donc fleuri. 
Localement, des travaux comme ceux d'Anne Tobé ${ }^{38}$ sur la commune de Passy sont exemplaires : ils concernent bien évidemment la célèbre église Notre-Dame-de-TouteGrâce du Plateau d'Assy, mais aussi l'ensemble de sanatoriums - architecture et décor- de la station climatique. Qui savait jusqu'ici que les cartons des vitraux de la chapelle du sanatorium de Praz-Coutant, réalisés par le peintre verrier de Lille Pierre Turpin en 1928, avaient été dessinés par un membre de la commission d'Art Sacré du diocèse de Lille, le chanoine Paul Pruvost? Dans ce contexte, il est évidemment exclu que la Cellule vitrail, à elle seule, puisse maîtriser l'inventaire exhaustif d'un si vaste corpus, mais elle peut se situer à un point de rencontre des actions menées à tous les niveaux. En la matière, les bénéfices d'une connaissance élargie de la production nationale et étrangère sont indispensables pour reconnaître dans l'océan de la production courante les verrières les plus significatives. Comment repérer, par exemple, les œuvres marquantes dans l'énorme production de l'atelier Max Ingrand ? $^{39}$. Cette ambition doit s'accompagner d'une méthode critique comparable à celle jusque-là appliquée au vitrail ancien. Ainsi, grâce à une véritable démarche de détective, un « intrus » a pu être identifié, qui s'était glissé à côté des auteurs affichés des célèbres vitraux de l'église Notre-Dame-de-la-Consolation du Raincy, Maurice Denis et Marguerite Huré : Jacques Gruber avait été sollicité en 1932 à la suite d'un conflit entre Marguerite Huré et le chanoine Félix Nègre, maître d'ouvrage. L'observation in situ des parties décoratives sans peinture des baies nord, qui témoignent d'un léger changement de parti dans l'exécution, confirme une source digne de foi, un article de Maurice Brillant dans la Vie catholique ${ }^{40}$.

Figure 18

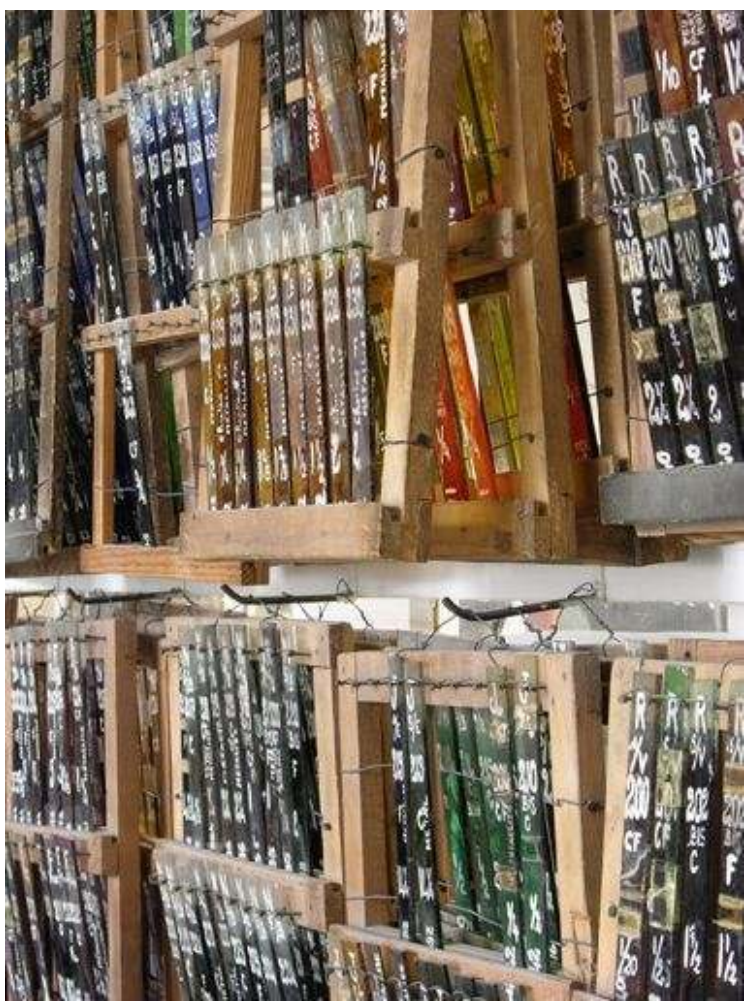

Reims (Marne), atelier Simon : palettes de verres

Phot. Inv. J. Philippot ( Inventaire général, ADAGP, 2005 
28

Cet exemple démontre l'intérêt évidemment primordial de conjuguer sources écrites et archéologie. Or les rencontres avec les acteurs de la vie artistique du XXe siècle ou avec leurs descendants en soulignent aussi les limites. Que de précisions, d'anecdotes ou de pistes nouvelles viennent au cours d'une conversation! Tout change quand l'œuvre d'art n'est plus seulement un objet bien classé, mais le résultat d'une démarche vivante. Les campagnes d'enquêtes orales lancées par la Cellule vitrail s'inscrivent ainsi dans une logique qui ne l'a jamais quittée : familière des œuvres, elle cherche à devenir l'intime de leurs auteurs (fig. $\left.\mathbf{n}^{\circ} \mathbf{1 8}\right)$.

\section{NOTES}

1. La collection nationale des ouvrages du Recensement comprend actuellement: Les vitraux de Paris, de Région parisienne, de Picardie et du Nord-Pas-de-Calais, vol. I. Paris : Editions du CNRS, 1978 ; Les vitraux du Centre et des Pays-de-la-Loire, vol. II. Paris : Editions du CNRS, 1981 ; Les vitraux de Bourgogne, de Franche-Comté et de Rhône-Alpes, vol. III. Paris : Editions du CNRS, 1986 ; Les vitraux de Champagne-Ardenne, vol. IV. Paris : Editions du CNRS, 1992 ; Les vitraux de Lorraine et d'Alsace, vol. V. Paris : CNRS-Editions, 1994 ; Les vitraux de Haute-Normandie, vol. VI. Paris : CNRS-Editions/Monum, Editions du patrimoine, 2001 ; Les vitraux de Bretagne, vol. VII. Rennes : Presses universitaires de Rennes, 2005.

2. Revue de l'Art, n 10, 1970 (en hommage à Jean Lafond, qui est également l'un des auteurs).

3. OTTIN, L. Le Vitrail. Son histoire, ses manifestations à travers les âges et les peuples. Paris : H. Laurens, s. d. (1896); MERSON, O. Les vitraux. Paris: Librairies-Imprimeries réunies, 1895 ; MAGNE, L. L'œuvre des peintres-verriers français. Paris : Firmin-Didot, 1885 et Les vitraux de Montmorency et d'Ecouen. Paris : Firmin-Didot, 1888 ; MALE, E. La peinture sur verre en France. Dans MICHEL, André. Histoire de l'art depuis les premiers temps chrétiens jusqu'à nos jours. t. I-2. Paris : Armand Colin, 1906, et t. II-4, Paris, 1911.

4. Les anciens clichés du service des Archives photographiques, peu à peu numérisés, sont rendus accessibles sur la base Mémoire: http://www.culture.gouv.fr/culture/inventai/presenta/ bddinv.htm

5. Revue de l'Art, n 10, 1970, p. 4 et 5.

6. Quelques-uns de ces trésors purent encore être montrés au Rijksmuseum d'Amsterdam en 1973, certaines reposes ayant tardé du fait de la multiplicité des chantiers.

7. Marcel Aubert, André Chastel, Louis Grodecki, Jean-Jacques Gruber, Jean Lafond, François Mathey, Jean Verrier et Jean Taralon. Paris : Editions des Deux-Mondes, 1958.

8. MALE, E. La peinture sur verre en France. Dans MICHEL, André. Histoire de l'art depuis les premiers temps chrétiens jusqu'à nos jours. t. I-2, Paris, 1906, p. 372.

9. Les notes de cet érudit, et ses photographies prises in situ ou en atelier, remontent pour certaines à 1908. Les cinq études qu'il a consacrées, de 1911 à 1973, au néerlandais Arnoult de Nimègue, un temps implanté à Rouen au début du XVIe siècle, témoignent des méthodes peu à peu développées.

10. AUBERT, M., GRODECKI, L., LAFOND, J., VERRIER, J. Les vitraux de Notre-Dame et de la SainteChapelle de Paris. Paris : Caisse nationale des Monuments historiques/CNRS, 1959 ; LAFOND, J. avec la collaboration de F. Perrot et P. Popesco. Les vitraux du chœur de l'église Saint-Ouen de Rouen. Paris : Caisse nationale des Monuments historiques/CNRS, 1970. 
11. Un tel dessein avait déjà fait l'objet de tentatives : Edouard Rayon avait parcouru pour le compte du service des Monuments historiques, entre 1909 et 1932, une dizaine de départements français, décrivant les vitraux à l'aide de croquis sommaires.

12. Après Anne Granboulan furent successivement engagées de 1972 à 1974 Laurence de Finance, Martine Callias Bey, Véronique Chaussé (David) et Françoise Gatouillat, formant l'équipe intégrée à l'Inventaire en 1979, placée sous la direction de Nicole Blondel. La «Cellule » a été rejointe en 1991 par Michel Hérold remplaçant Laurence de Finance.

13. Voir le site $\mathrm{du}$ Ministère: http://www.culture.gouv.fr/culture/inventai/presenta/ bddinv.htm

14. Les vitraux de Basse-Normandie seront bientôt publiés (volume VIII).

15. Cf. KORN, U.-D. Licht, Glas, Farbe. Aix-la-Chapelle : M. Brimberg, s. d. (1990), p. 157.

16. En Angleterre, aux Pays-Bas et aux Etats-Unis.

17. L'Aquitaine, l'Auvergne, le Languedoc-Roussillon, le Limousin, Midi-Pyrénées, PoitouCharentes et Provence-Alpes-Côte d'Azur feront l'objet de deux volumes supplémentaires, le dernier comprenant un index cumulé.

18. Cf. CALLIAS-BEY, M. et de FINANCE, L. Les bénéfices d'un inventaire : premières réflexions autour des chiffres. Mémoire de verre. Vitraux champenois de la Renaissance. Châlons-surMarne, 1990, p. 25-56 (coll. Cahiers de l'Inventaire, $\mathrm{n}^{\circ} 22$ ).

19. Recensement, vol. III, 1986, p. 138-139 et fig. 119 ; GATOUILLAT, F. Vitreries de type cistercien dans l'Yonne. Actes du 56e Congrès de l'Association bourguignonne des Sociétés savantes. 1985, p. 59-64.

20. Recensement, vol. III, 1986, p. 16, 135 et pl. VII.

21. Recensement, vol. V, 1994, p. 239 et p. 196 ; GATOUILLAT, F. Un panneau de l'église SainteMadeleine de Strasbourg entre au Musée de l'đEuvre-Notre-Dame. Cahiers alsaciens d'Archéologie d'Art et d'Histoire, t. XXXVII, 1994, p. 141-145; id., Les vitraux anciens du Séminaire de Caen. Annales de Normandie, $n^{\circ}$ 51, 2001, p. 121-138.

22. Recensement, vol. III, 1986, p. 54 et fig. 37 ; GATOUILLAT, F., LEPROUX, G.-M., PILLET, E. L'église Saint-Merry de Paris : un monument daté par ses vitraux. Cahiers de la Rotonde, $\mathrm{n}^{\circ} 19, \mathrm{p}$. 47-114 (p. 58, 61, 64) ; GATOUILLAT, F. La grande verrière occidentale de Saint-Merry de Paris et ses auteurs : un cartonnier néerlandais au service d'ateliers parisiens. Revue de l'Art, $\mathrm{n}^{\circ} 147$, 2005/1, p. 69-78.

23. LAFOND. La Renaissance. Le vitrail français, 1958, p. 215.

24. PERROT, F. Le vitrail à Rouen. Rouen, 1972, p. 36.

25. CALLIAS BEY, M., CHAUSSE, V., HEROLD, M. Vitraux retrouvés de l'église Saint-Vincent de Rouen. Rouen, 1995 (catalogue de l'exposition, Musée des Beaux-Arts de Rouen, 10 déc. 1995-26 février 1996).

26. HEROLD, M. A propos du « Maître de la vie de saint Jean-Baptiste » : recherches sur l'usage du patron à grandeur au début du xvie siècle. Vitrail et arts graphiques (XVe-XVIe s.), Cahiers de l'Ecole du patrimoine, $\mathrm{n}^{\circ}$ 4, 1998, p. 48-60; id., La production normande du Maître de la vie de saint Jean-Baptiste. Nouvelles recherches sur l'usage des documents graphiques dans l'atelier du peintre-verrier à la fin du Moyen Age. Pierre, lumière, couleur. Etudes d'histoire de l'art du Moyen Age en l'honneur d'Anne Prache, F. Joubert et D. Sandron éd. Paris : Presses universitaires de la Sorbonne, 1999, p. 469-485.

27. HEROLD, M. Aux sources de «l'invention »: Gaultier de Campes, peintre à Paris au début du XVIe siècle. Revue de l'Art, n 120, 1998-2, p. 49-57 ; LEPROUX, G.-M. La peinture à Paris sous le règne de François $\mathrm{I}^{\mathrm{er}}$. Paris : Presses universitaires de la Sorbonne, 2001 (coll. Corpus vitrearum, Etudes IV), p. 39-108.

28. A Saint-Léonard de Tamsweg en Autriche, à Zetting en Moselle (vers 1440-1450), comme à la cathédrale de Berne (milieu du XVe siècle), dans les vitraux dispersés de l'église des Carmélites de Boppard-am-Rhein (vers 1440), et sur un panneau de Bad-Urach (vers 1475), d'origine 
strasbourgeoise comme celui des Tentations du Christ conservé au musée des Beaux-Arts d'Arras (vers 1460-1470) ; cf. GATOUILLAT, F. Les performances techniques des ateliers strasbourgeois : nouvel aperçu sur l'apparition de la gravure à l'acide et des émaux. Corpus Vitrearum Medii Aevi XIXth international Colloquium Proceedings. Cravovie, 1998, p. 103-111.

29. Les raisons en sont multiples, aujourd'hui assez bien cernées à la lecture des archives : ces reprises sont le fruit de l'émulation entre les commanditaires, désireux d'offrir des verrières «telles et pareilles que celles de... » tel édifice ; elles s'expliquent aussi par la tentation qu'a le peintre verrier d'exploiter le fonds d'atelier qu'il s'est constitué.

30. ZANARDI, B. Projet dessiné et «patrons » dans le chantier de la peinture murale au Moyen Age. Revue de l'Art, n 124, 1999/2, p. 43-55.

31. Des dossiers, inédits dans le Recensement, ont alors été constitués, intégrés à la documentation des services régionaux de l'Inventaire.

32. La première publication de ce répertoire figure dans la Revue de l'Art, $n^{\circ} 72,1986$.

33. Voir le site : http://www.culture.gouv.fr/documentation/marque/orfeo-frame.htm

34. MIGNOT, C. L'architecture au XIXe siècle. Fribourg: Office du Livre, 1983, p. 7.

35. Par notre action ont en revanche récemment pu être sauvés de la dispersion ou de la destruction le fonds de l'atelier de Marguerite Huré et de Marcelle Lecamp, déposé au Musée des Années 30 à Boulogne-Billancourt, et celui de l'atelier Bony, conservé aux Archives du Monde du travail à Roubaix.

36. Au rang desquelles l'Inventaire, certaines universités et l'EPHE, où les travaux de recherche se sont multipliés sur cette thématique, ou des institutions telles que le Grimco (groupe de recherche en iconographie moderne et contemporaine créé en 1984 par C. Brisac avec l'université du Maine). Des publications témoignent de cet intérêt croissant ; voir par exemple : CHAUSSE, V. Verrières du XXe siècle en Haute-Normandie. Rouen, 1996 (coll. Itinéraires du patrimoine, $\left.\mathrm{n}^{\circ} 128\right)$.

37. L'ouvrage de Laurence de FINANCE. Un patrimoine de lumière, 1830-2000 (coll. Cahiers du Patrimoine, $n^{\circ}$ 67), Paris, 2003, est à ce titre exemplaire.

38. Les recherches d'Anne Tobé, présidente du CREHA, sont en partie publiées dans la revue Vatusium, $n^{\circ} 4,2001$, p. 30-38.

39. On peut notamment considérer comme exceptionnels les vitraux civils que Max Ingrand a réalisés à ses débuts, tel le décor de l'immeuble construit par l'architecte Jean Desbouis en 1932 au 116bis, Avenue des Champs-Elysées.

40. DAVID, V. De l'espoir retrouvé et des vitraux du Raincy. Dans Laurence de FINANCE. Un patrimoine de lumière, 1830-2000 (coll. Cahiers du Patrimoine, nº 67), Paris, 2003, p. 275-285.

\section{RÉSUMÉS}

Le Recensement des vitraux anciens de la France sera bientôt achevé, conduit dans le cadre du ministère de la Culture depuis 1972 et intégré aux missions de l'Inventaire général depuis 1979. Cet inventaire méthodique et exhaustif est régulièrement publié dans des ouvrages conçus comme des instruments de travail qui visent à apporter au monde de la recherche une documentation précise, critique et présentée avec objectivité, sur toutes les œuvres de ce domaine patrimonial, sans hiérarchie. Ce recensement fait émerger une multiple et inépuisable matière à réflexion, qui alimente des problématiques communes à d'autres disciplines de 
l'histoire de l'art (usages techniques, questions de la commande artistique, de la diffusion des formes, etc.). Un retour sur la documentation accumulée et sur les travaux qui se sont ajoutés au fil de l'expérience permet d'apprécier l'évolution du champ scientifique que constitue le vitrail, qui a beaucoup gagné en participant d'une vision élargie.

Soon will be completed the Recensement des vitraux anciens de la France, France's general inventory of stained-glass windows from the Middle Ages to the end of the Ancien Régime, which has been carried out since 1972 under the auspices of the Ministry of Culture, in charge since 1979 of the Inventaire général. The results of this methodical and exhaustive inventory are published at regular intervals in works which are designed as tools for research, providing precise critical documentation covering all the works in this particular field of the national heritage, objectively and without hierarchical judgement. The survey work has drawn attention to stained-glass windows as an extremely rich and varied source, informing problematics shared by other disciplines in art history (technical usages, problems relating to artistic patronage, to the diffusion of forms, etc.) An overview of the documentation accumulated over the years and of a certain number of research projects inspired by the inventory experience leads to an evaluation of the scientific domain of stained-glass, a domain which has developed considerably thanks to the broadening vision within which it is now studied.

\section{INDEX}

Keywords : vitrail, recensement, inventaire, vitraux anciens, verrière, peinture sur verre, patrimoine, sauvegarde, Jean Lafond, monuments historiques, cathédrale, patrimoine verrier, Louis Grodecki, Chartres, Bourges, restauration, laboratoire de recherche sur le patrimoine français, corpus vitrearum, Bretagne, Normandie, Champagne, collections privées, Arnoult de Nimègue, Rouen, Jean Barbe, Gaillon, Amboise, Paris, verre, émaux, grisaille, peintre cartonnier, peintre verrier, Louis Barillet, Jacques Gruber, Max Ingrand, Marguerite Huré, enquête orale, datation

\section{AUTEURS}

\section{FRANÇOISE GATOUILLAT}

Ingénieur d'études. Direction de l'architecture et du patrimoine. Centre André Chastel, UMR 8150. francoise.gatouillat@paris4.sorbonne.fr

\section{MICHEL HÉROLD}

Conservateur du patrimoine. Direction de l'architecture et du patrimoine. Centre André Chastel, UMR 8150. michel.herold@paris4.sorbonne.fr

\section{VÉRONIQUE DAVID}

Ingénieur d'études. Direction de l'architecture et du patrimoine. Centre André Chastel, UMR 8150.veronique.david@paris4.sorbonne.fr 